\title{
Não linearidade entre câmbio e preços no Brasil e implicações para uma estratégia de desenvolvimento econômico
}

\author{
Non-linearity between exchange and prices in Brazil and \\ implications for an economic development strategy
}

\author{
MATEUS RAMALHO RIBEIRO DA FONSECA* \\ ELIANE CRISTINA DE ARAÚJO** \\ ELISANGELA ARAÚJO***
}

RESUMO: O objetivo deste artigo é realizar uma análise da política monetária no Brasil a partir de um modelo de Vetores Autorregressivos com Cadeias de Markov (MS-VAR), na busca de evidências da não linearidade da relação entre câmbio e preços no Brasil. A análise demonstrou que, em períodos de apreciação cambial, tanto pelo lado da demanda quanto da oferta, existe um conjunto de forças que determinam uma trajetória baixista para os níveis de preços, sugerindo que a taxa de câmbio tem um papel fundamental no controle da inflação. Contudo, se aponta a necessidade de reavaliação do papel do câmbio no Brasil.

PALAVRAS-CHAVE: Taxa de câmbio; inflação; economia brasileira; Desenvolvimento econômico.

ABSTRACT: The objective of this article is to perform an analysis of monetary policy in Brazil, using a Markov Chain Autoregressive Vector (MS-VAR) model, in the search for evidence of non-linearity in the relationship between exchange and prices in Brazil. The analysis showed that in periods of exchange appreciation, both on the demand side and the supply side, there is a set of forces that determine a downward trajectory for price levels, suggesting that the exchange rate plays a fundamental role in the control of inflation. However, there is a need to reassess the role of the exchange rate in Brazil.

KEYWORDS: Exchange rate; inflation; Brazilian economy; development economy. JEL Classification: E44; E52; O23.

\footnotetext{
* Doutor em Economia - Área de Concentração: Economia do Desenvolvimento - Programa de Pós-Graduação em Economia da Universidade Federal do Rio Grande do Sul (PPGE/UFRGS), Porto Alegre/ RS, Brasil. E-mail: mateusramalho88@hotmail.com.

* Professora Associada da Universidade Estadual de Maringá-UEM e pesquisadora do CNPq, Maringá/PR, Brasil. E-mail: elianedearaujo@gmail.com.

*** Professora Adjunta da Universidade Estadual de Maringá - UEM, Maringá/PR, Brasil E-mail: elisangela15.araujo@gmail.com. Submetido: 30/Agosto/2017; Aprovado: 10/outubro/2018.
} 


\section{INTRODUÇÃO}

Dentre os diversos canais de transmissão da política monetária, a taxa de câmbio se destaca como um dos mais importantes, o que tem sido amplamente documentado na literatura econômica brasileira nas últimas décadas.

De fato, após a mudança para o regime de câmbio flutuante e a respectiva introdução do Regime de Metas de Inflação (RMI) no Brasil em meados de 1999, a taxa de câmbio apresentou considerável variabilidade chegando a ultrapassar R \$ 4,00, como em 2002 e 2015, por exemplo, ou ainda, situar-se próximo de R \$1,50, como ocorrido nos anos de 2007 e em 2011. Seja em resposta aos acontecimentos domésticos - com destaque para as alterações na taxa básica de juros no cumprimento das metas de inflação - seja em razão de choques externos, alteração das percepções sobre os rumos da economia geralmente implicaram maior ou menor disposição à entrada de capitais estrangeiros, especialmente, seja os de curto prazo, contribuindo para a volatilidade deste preço fundamental da economia brasileira ${ }^{1}$.

A questão a ser destacada, contudo, é que essas diferentes trajetórias da taxa de câmbio têm um papel central para explicar a variação dos preços domésticos. Todavia, a despeito das consequências da variação cambial ser um tema de interesse, em particular, a estimação do chamado coeficiente de repasse cambial, ou pass-through da taxa de câmbio, que mede os efeitos de mudanças na cotação da moeda doméstica sobre o nível de preços, bem menos atenção tem sido dada ao fenômeno da não linearidade, isto é, de como diferentes níveis da moeda doméstica (apreciação/depreciação) interferem na trajetória da inflação no país.

A não linearidade, nesse sentido, lança luz a questões mais complexas, que podem ajudar na compreensão da condução da política macroeconômica doméstica e seus principais resultados, que passa a ter na taxa de câmbio uma aliada (ou um obstáculo) no cumprimento das metas estipuladas pelas Autoridades Monetárias. A constatação desse fenômeno faz com que a discussão avance para uma seara mais ampla, abarcando questões como a da importância da adoção de mecanismos de gerenciamento da taxa de câmbio, não somente com o objetivo de manter a inflação dentro da meta, mas, sobretudo, para situar esse "preço" fundamental em níveis condizentes com as necessidades de uma economia em desenvolvimento, como é o caso do Brasil.

Sobre essa discussão vale ressaltar, inclusive, que nos últimos anos, particularmente após a crise financeira internacional deflagrada em 2008, diversas economias - desenvolvidas e em desenvolvimento - convencidas dos limites de algumas políticas macroeconômicas tidas como consensuais pelo mainstream para a promoção do crescimento e estabilidade, como é o caso da liberalização financeira, voltaram-se para a adoção de mecanismos disciplinadores dos fluxos internacionais de capitais, de modo a se promover crescimento econômico com estabilidade ${ }^{2}$. O Brasil,

\footnotetext{
${ }^{1}$ Ver Bresser-Pereira et al. (2014).

${ }^{2}$ Ver Akerlof et al. (2014).
} 
todavia, continuou apartado deste debate, mantendo intactos os fundamentos que conduzem a política macroeconômica, desde 1999.

À luz dessa constatação, o objetivo deste artigo é realizar uma análise empírica da condução da política monetária com base em um modelo Markov Swichting Vector Autoregressive (MS-VAR) ou Vetores Autorregressivos com Cadeias de Markov, na busca de evidências da existência de não linearidade da relação entre câmbio e preços no Brasil. O intuito é verificar se existem mudanças de regime na política monetária e se, em razão destas, decorrem comportamentos distintos da economia. A ideia é investigar ainda se, decorrente da tal não linearidade, variáveis centrais para a condução da política monetária - taxa de juros, taxa de câmbio, inflação e nível de atividade econômica - estão sujeitas a mudanças de regime desde a adoção do RMI em 1999.

A pesquisa se encontra estruturada da seguinte forma: após esta introdução, a segunda seção apresenta uma discussão teórica e também estatísticas descritivas da relação entre câmbio e preços na economia brasileira a partir do RMI. Na terceira seção é apresentada a metodologia e a base de dados utilizada para a investigação da hipótese da não linearidade da relação entre câmbio e preços no Brasil, por meio dos modelos Markov Swichting Vector Autoregressive (MS-VAR). Na sequência, a quarta seção traz as estimações e discute os resultados encontrados. $\mathrm{Na}$ quinta seção, faz-se uma discussão sobre a condução da política cambial no Brasil, bem como as relações entre câmbio e inflação e câmbio e desenvolvimento econômico. Finalmente, são apresentadas as considerações finais do trabalho, adiantando-se que as evidências da não linearidade sugeridas pela presente pesquisa apontam para a necessidade de uma reavaliação da política macroeconômica que vem sendo adotada no Brasil, com destaque para os efeitos sobre a trajetória da taxa de câmbio, como um dos obstáculos ao crescimento e a estabilidade nas últimas décadas.

\section{RELAÇÃO ENTRE CÂMBIO E INFLAÇÃO: ASPECTOS TEÓRICOS E EVIDÊNCIAS PARA A ECONOMIA BRASILEIRA}

O interesse pelo estudo da relação taxa de câmbio e inflação ganhou alento a partir do início dos anos 1970, quando ocorreu o fim do sistema de paridade das taxas de câmbio em relação ao dólar, fixadas pelo acordo de Bretton Woods (1944), com a respectiva adoção do sistema de taxas de câmbio flutuantes por diversos países (MENON, 1995).

$\mathrm{Na}$ medida em que as flutuações da taxa de câmbio não possibilitaram o restabelecimento do equilíbrio do balanço de pagamentos, mas levavam a novos desequilíbrios, tornou-se crescente a busca de alternativas para os novos problemas econômicos, com destaque para o estudo da relação entre a taxa de câmbio e o nível de preços, em especial, dos bens comercializados internacionalmente (tradeables) com vistas à implementação de políticas econômicas mais assertivas.

Voltando-se o olhar especificamente para a economia brasileira, esta relação assume grande importância, em particular, após a mudança para o regime de câmbio flu- 
tuante e a respectiva introdução do Regime de Metas de Inflação (RMI). Desde então, a taxa de câmbio tem sido um dos mecanismos relevantes pelos quais a política monetária e seu instrumento principal (a taxa de juros básica Selic) afeta o nível geral de preços, sendo a investigação deste tema uma constante na literatura econômica brasileira (Minella \& Correa, 2005; Tombini \&Alves, 2006, Belaisch, 2003).

Ao efeito que a taxa de câmbio exerce sobre a inflação denomina-se pass-throu$g h$, ou repasse cambial para os preços domésticos, e mede a variação percentual da taxa de inflação ante a variação de $1 \%$ na taxa de câmbio. Esse repasse pode ser: i) nulo, se variações cambiais não são repassadas aos preços; ii) completo, ou igual a um, se variações cambiais são totalmente repassadas aos preços e iii) entre zero e um, se apenas parte da variação do câmbio é repassado aos preços domésticos ${ }^{3}$.

A razão para tal influência decorre de que a variação da cotação da moeda estrangeira acarreta diversos efeitos, diretos e indiretos, sobre a inflação. Como efeito direto, tem-se a mudança dos preços dos bens que são comercializáveis no mercado internacional (os bens tradeables). Indiretamente, o câmbio afeta a inflação de duas formas: i) via preço das matérias-primas importadas na elaboração de bens domésticos - quando a taxa de câmbio sofre apreciação (depreciação), o custo de produção desses bens diminui (aumenta), o que implica provável redução (aumento) no preço; e ii) substituição de bens domésticos por similares importados - quando o câmbio aprecia, o preço dos bens importados diminui. Esse efeito acarreta um deslocamento de parte da demanda doméstica para as importações, tendo por resultado a queda na demanda agregada e, com isso, menor pressão sobre o nível de preços.

A relação câmbio-preços é apresentada na Tabela 1 na qual é possível constatar a considerável variabilidade da taxa de câmbio nominal Real/US\$ ao longo do período 1999-2016. Tomando-se como exemplo os últimos seis anos (2011-2016), pode-se verificar que a referida taxa oscilou entre $\mathrm{R} \$ 1,67$ (2011) e R \$ 3,33 (2015) em média, embora, em termos nominais, a cotação da moeda estrangeira ultrapassou os R \$ 4,00 em alguns períodos, bem como se aproximou de R \$ 1,50 em outros, evidenciando uma excessiva volatilidade cambial. Essas oscilações podem ser relacionadas ao comportamento da inflação no período, mais especificamente, ao próprio desempenho do RMI, como indicado na Tabela 1.

Com referência à trajetória da inflação no Brasil, medida pelo Índice de Preços ao Consumidor Amplo (IPCA), a partir da introdução do RMI em 1999, nota-se que os limites superiores das metas estipuladas foram ultrapassados em 4 anos (2001, 2002, 2003 e 2015) por uma margem substancial, especialmente em 2002 e 2015. Além disso, cabe mencionar que no ano de 2004 a meta de inflação só foi atingida após a revisão da meta para cima e a consequente redefinição dos referidos intervalos, evidenciando a importância da taxa de câmbio no comportamento dos preços domésticos.

\footnotetext{
${ }^{3}$ Confira Kannebley Jr. (2000).
} 
Tabela 1: Inflação (IPCA), metas e intervalos de tolerância

e taxa de câmbio nominal, Brasil, 1999-2016

\begin{tabular}{|c|c|c|c|c|}
\hline Ano & $\begin{array}{l}\text { Meta de } \\
\text { inflação }\end{array}$ & $\begin{array}{l}\text { Intervalo de } \\
\text { tolerância }\end{array}$ & $\begin{array}{l}\text { Inflação efetiva } \\
\text { (IPCA) }\end{array}$ & $\begin{array}{c}\text { Taxa de câmbio } \\
\text { R\$/US\$1 }\end{array}$ \\
\hline 1999 & 8 & $6-10$ & 8,94 & 1,81 \\
\hline 2000 & 6 & $4-8$ & 5,97 & 1,83 \\
\hline 2001 & 4 & $2-6$ & 7,67 & 2,35 \\
\hline 2002 & 3,5 & $1,5-6,5$ & 12,53 & 2,92 \\
\hline 20032 & 4 & $1,5-6,5$ & 9,3 & 3,08 \\
\hline 2004 & 5,5 & $3,5-8$ & 7,6 & 2,93 \\
\hline 2005 & 4,5 & $2,5-7,5$ & 5,69 & 2,43 \\
\hline 2006 & 4,5 & $2,5-7,5$ & 3,14 & 2,18 \\
\hline 2007 & 4,5 & $2,5-6,5$ & 4,46 & 1,95 \\
\hline 2008 & 4,5 & $2,5-6,5$ & 5,9 & 1,83 \\
\hline 2009 & 4,5 & $2,5-6,5$ & 4,31 & 2,00 \\
\hline 2010 & 4,5 & $2,5-6,5$ & 5,91 & 1,76 \\
\hline 2011 & 4,5 & $2,5-6,5$ & 6,5 & 1,67 \\
\hline 2012 & 4,5 & $2,5-6,5$ & 5,84 & 1,95 \\
\hline 2013 & 4,5 & $2,5-6,5$ & 5,91 & 2,16 \\
\hline 2014 & 4,5 & $2,5-6,5$ & 6,41 & 2,35 \\
\hline 2015 & 4,5 & $2,5-6,5$ & 10,67 & 3,33 \\
\hline 2016 & 4,5 & $2,5-6,5$ & 6,3 & 3,25 \\
\hline
\end{tabular}

Notas: 1. Média do período, cotação para a venda. 2. A carta aberta de 21/03/2003 revisou as metas para 8,5 em 2003 e 5,5 em 2004, respectivamente. Fonte: BCB (2017a).

Com respeito aos resultados positivos, pode-se constatar que a inflação esteve dentro do intervalo de tolerância da meta em 12 anos (1999, 2000, 2004, 2005, 2006, 2007, 2009, de 2011 a 2014 - momento em que se situou muito próximo ao limite de tolerância superior - e 2016), sendo que, em apenas três $\left(2000,2006\right.$ e 2007) foi inferior ao centro da meta ${ }^{4}$. Vale notar que, em 2006, foi alcançado o menor IPCA anual desde o início da série histórica, e mais ainda, que o único período no qual a inflação esteve realmente baixa foi a fase entre 2006-2009, excluindo-se o ano da crise de 2008, momento em que a taxa de câmbio assumiu uma tendência de apreciação continuada, conforme pode ser visualizada no Gráfico 1.

\footnotetext{
${ }^{4}$ É importante destacar que a inflação brasileira, sob o RMI, se manteve em níveis relativamente altos (em média 6,83\% a.a.) se comparado à média mundial, que foi de 4,07\% entre 1999 e 2016 (IMF, 2017).
} 
Figura 1: Taxa de câmbio (R\$/US\$) e inflação (IPCA) no Brasil (1999-2017)

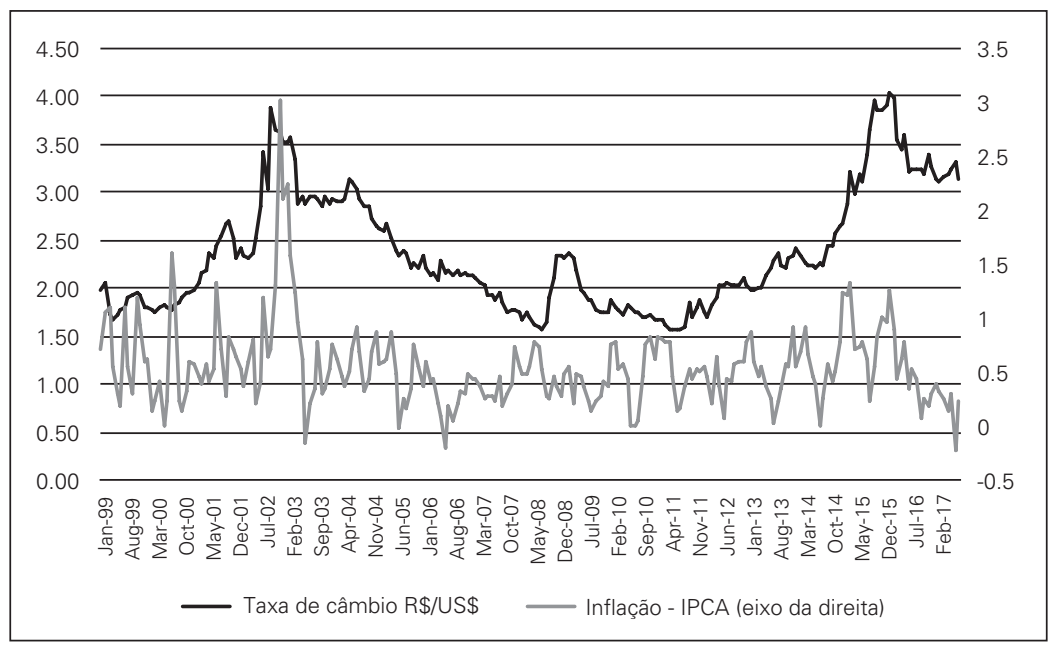

Fontes: BCB (2017a) e IBGE (2017).

A análise do Gráfico 1 evidencia que a trajetória da taxa de câmbio e dos preços no Brasil apresenta uma relação direta, de modo que, nas fases em que ocorreu um aumento da referida taxa (depreciação cambial), observou-se um salto dos preços, como exemplo, nos anos de 2001, 2002 e 2003, que foram marcados pelo efeito-contágio da crise da Argentina e das eleições presidenciais brasileiras. Da mesma forma, no último trimestre de 2008, a crise do subprime e, mais recentemente, entre 2013 e 2016, foram fatores externos como o fim das políticas de quantitative-easy promovido por alguns países desenvolvidos, aliado à crise política e econômica brasileira, que estiveram entre os principais fatores explicativos da alta da taxa de câmbio e a consequente elevação da inflação. Diferentemente, em fases de queda da taxa de câmbio, a inflação apresentou-se relativamente mais baixa, como por exemplo entre 2005 e 2009, com exceção do ano de 2008, como já mencionado 5 . Parece plausível, portanto, a crença de que a Autoridade Monetária, ainda que de forma implícita, leva em conta o comportamento da taxa de câmbio quando toma decisões com respeito à taxa de juros básica no Brasil.

Além da influência direta e indireta que o câmbio exerce sobre os preços, outra questão central, mas que tem recebido menos importância, se refere aos diferentes impactos que a taxa de câmbio exerce sobre os preços em momentos distintos, de depreciação ou apreciação da moeda. Isto é, pode ser que o repasse cambial ocor-

\footnotetext{
${ }^{5}$ Tendo em vista a importância do câmbio para a inflação, alguns autores incluíram a variação da taxa de câmbio na estimação da função de reação do Banco Central, que explicitamente considera apenas o hiato de inflação e o hiato do produto. $\mathrm{O}$ argumento utilizado é que esta variável é importante para explicar variações nas taxas de juros em países emergentes, que são mais suscetíveis a choques externos. Minella et al. (2003), por exemplo, estimaram um modelo autorregressivo vetorial (VAR) e concluíram que os choques na taxa de câmbio explicam uma parte significativa dos erros de juros e inflação.
} 
ra de forma não linear e, em assim sendo, seria relevante conhecer as implicações que tal fato poderia ter.

Neste sentido, com base na Figura 1, pode-se dividir o período 1999-2017 em seis subperíodos, conforme o comportamento da taxa de câmbio, a saber: i) janeiro de 1999 a setembro de 2002 (depreciação); ii) outubro de 2002 a julho de 2008 (apreciação); iii) agosto de 2008 a fevereiro de 2009 (depreciação) iv) março de 2009 a julho de 2011 (apreciação); v) agosto de 2012 a janeiro de 2016 (depreciação); e vi) fevereiro de 2016 a fevereiro de 2017 (apreciação). Esta análise é evidenciada na Tabela 2, que mostra os episódios de apreciação/depreciação da taxa de câmbio ocorridos desde a implementação do RMI no Brasil, além da correlação entre câmbio e inflação nos diferentes subperíodos.

Tabela 2: Câmbio e inflação no Brasil, 1999-2017

\begin{tabular}{c|c|c}
\hline Período & Episódio & Correlação câmbio/inflação \\
\hline $1999: 1$ a 2002:9 & Depreciação & 0,1373 \\
\hline $2002: 10$ a $2008: 7$ & Apreciação & 0,5654 \\
\hline $2008: 8$ a $2009: 2$ & Depreciação & 0,5754 \\
\hline $2009: 3$ a 2011:7 & Apreciação & $-0,2151$ \\
\hline $2011: 8$ a 2016:1 & Depreciação & 0,4818 \\
\hline $2016: 2$ a 2017:2 & Apreciação & 0,7442 \\
\hline
\end{tabular}

Fonte: BCB (2017a).

Conforme a Tabela 2, houve três subperíodos de depreciação (janeiro/1999 a setembro/2002, agosto/2008 a fevereiro/2009 e agosto/2011 a janeiro/2016). No primeiro e no último subperíodos, a elevação da cotação da moeda estrangeira se fez acompanhar por inflação mais elevada. Vale notar, inclusive, que nesses subperíodos considerados a meta de inflação não foi alcançada em três anos $(2001,2002$ e 2015).

Já no segundo subperíodo de depreciação, que durou apenas 6 meses e esteve diretamente relacionado aos efeitos da crise norte-americana deflagrada no segundo semestre de 2008 (agosto/2008 a fevereiro/2009), nota-se que a inflação se manteve em queda ${ }^{6}$, a despeito da elevação do câmbio. Vale mencionar, porém, que nesta fase a taxa de câmbio se elevou, mas continuou situando-se em patamares consideravelmente baixos (entre $\mathrm{R} \$ 1,63$ e $\mathrm{R} \$ 2,37$ ), evitando pressão sobre a inflação, que em média situou-se bem próxima da meta estipulada. Destaque-se ainda o resultado encontrado por Araújo e Modenesi (2010), o qual sugere que as pressões da depreciação cambial sobre os preços foram amenizadas nesse período

\footnotetext{
${ }^{6}$ Sobre os fatores que evitaram o aumento dos preços em 2008 e 2009 destacam-se, além do fato da taxa de câmbio a queda dos preços das commodities, o que no Brasil, por exemplo, resultou em queda do preço da gasolina e de outras matérias-primas e/ou insumos básicos (BCB, 2017b).
} 
por choques de demanda negativos e choques de oferta positivos que puxaram os preços para baixo.

Analisando agora os subperíodos de apreciação cambial, é possível notar que a inflação caiu, relativamente à trajetória prévia, em duas das três fases (outubro/2002 a julho/2008 e fevereiro/2016 a fevereiro/2017). No primeiro período seguiu tendência declinante, caindo aos menores níveis desde a adoção do RMI e, inclusive, alcançou seu valor histórico mais baixo desde 2006. No outro subperíodo, que abrangeu fevereiro de 2016 a fevereiro de 2017, a taxa de câmbio, que havia superado os $\mathrm{R} \$ 4,00$ nos meses precedentes, recuou para próximo dos $\mathrm{R} \$$ 3,10 , o que, aliado à forte retração da economia brasileira, trouxe a inflação para $5,1 \%$ a.a., em média, no período. A exceção foi o período entre março/2009 e julho/2011 quando, a despeito da apreciação, a inflação obteve ligeira elevação, embora esta variável tenha ficado bem próximo da meta (4,8\% a.a., em média).

Um último aspecto a destacar com respeito à Tabela 2 , se refere à correlação entre taxa de câmbio e inflação. Nota-se que esta correlação foi baixa somente no primeiro subperíodo de depreciação $(0,13)$ e alta nos dois subperíodos de depreciação seguintes $(0,57$ e 0,48$)$. Por outro lado, nos períodos de apreciação, deixando-se de lado o período do imediato pós-crise, nota-se que esta correlação foi elevada: de 0,56 e 0,74 , sugerindo que a cotação da moeda estrangeira pode auxiliar no controle da inflação.

Tal análise esclarece que o papel da taxa de câmbio é relevante na determinação dos preços no Brasil e, sobretudo, nos períodos em que a moeda se encontra em trajetória de apreciação. Neste sentido, se a taxa de câmbio é baixa o suficiente, é mais plausível a inflação se apresentar em patamares mais baixos, sendo possível à Autoridade Monetária alcançar o objetivo de cumprir a meta de inflação sem maiores dificuldades veja-se por exemplo o período (2005-2009). Essa ideia da existência de uma possível não linearidade da política monetária é investigada a seguir.

\section{METODOLOGIA E BASE DE DADOS}

O modelo empregado no presente estudo é o MS-VAR (Vetores Autorregressivos com Cadeias de Markov). Esse modelo permite estimações econométricas em séries temporais com mudanças de regime, isto é, permite a análise de variáveis macroeconômicas de maneira não linear.

Segundo Krolzig $(1996,1997,1998)$ os modelos MS-VAR surgiram de dois instrumentais importantes: os VAR, ou vetores autorregressivos, surgiram com Sims (1990) e hoje são muito utilizados para analisar as variáveis macroeconômicas; e de modelos que se utilizam das cadeias markovianas para analisar as mudanças de regime em variáveis macroeconômicas ${ }^{7}$.

Krolzig (1997) criou uma notação simples que permite identificar os modelos

\footnotetext{
${ }^{7}$ Diversos autores têm trabalhado com este modelo apontando a existência de não linearidade nos modelos macroeconômicos. Para maiores detalhes ver: Hamilton (1989); Krolzig (1997); Ehrmann, Ellison e Valla, (2003).
} 
com os parâmetros sendo ou não dependentes do regime e que é expressa de maneira simplificada na Tabela 3:

Tabela 3: Tipos de modelo MS-VAR segundo os parâmetros

\begin{tabular}{c|c|c|c|c|c}
\hline Parâmetros & $\begin{array}{c}\operatorname{MSM}(m)- \\
\operatorname{VAR}(p)\end{array}$ & $\begin{array}{c}\operatorname{MSMH}(m)- \\
-\operatorname{VAR}(p)\end{array}$ & $\begin{array}{c}\operatorname{MSI}(m)- \\
-\operatorname{VAR}(p)\end{array}$ & $\begin{array}{c}\operatorname{MSIH}(m)- \\
\operatorname{VAR}(p)\end{array}$ & $\begin{array}{c}\mathrm{MSIAH}(m)- \\
-\operatorname{VAR}(p)\end{array}$ \\
\hline$\mu$ & variante & variante & - & - & - \\
\hline$v$ & - & - & variante & variante & variante \\
\hline$\Sigma$ & invariante & variante & invariante & variante & variante \\
\hline $\mathrm{A}_{\mathrm{i}}$ & invariante & invariante & invariante & invariante & variante \\
\hline
\end{tabular}

Fonte: Krolzig (1997). Elaboração própria.

Notação: MSM: cadeias markovianas em que a média varia; MSMH: cadeias markovianas em que a média e na variância variam; MSI: cadeias markovianas em que o intercepto varia; $\mathrm{MSIH}$ : cadeias markovianas em que o intercepto e a variância variam; MSIAH: cadeias markovianas em que o intercepto, os parâmentos autorregressivos e a variância variam; $m$ : número de regimes; $p$ : número de defasagens do VAR; $\mu$ : média $(M), v$ : intercepto (I), $\Sigma$ : variância $(H), A_{i}$ : parâmetros autorregressivos (A).

Neste trabalho, será usado o MS-VAR com todos os parâmetros dependentes do regime, o que, segundo a Tabela 3, daria um modelo $\operatorname{MSIAH}(m)-\operatorname{VAR}(p)$ e o método de estimação para esse modelo (MS-VAR) é o algoritmo de Maximização Expectacional ou Expectation-Maximization $(\mathrm{EM})^{8}$. Como não foi realizado nenhum tratamento dos dados, optou-se por estimar, propositadamente, um modelo em que tanto o intercepto, como os parâmetros autorregressivos, quanto a variância variem como forma de flexibilização da estimação e regressão do modelo. Sem essa flexibilização, o modelo se tornaria mais restrito e de difícil fechamento para sua estimação.

Há de se lembrar que há nos dados a presença de mudanças bruscas nas variáveis analisadas, principalmente nos anos de 2001, 2002, 2007 e 2008, que inviabilizam qualquer forma de estimação por método linear. Dessa forma, a opção mais viável é estimar um modelo que endogeniza essas mudanças bruscas (também conhecidas como quebras estruturais na estatística), que no caso seria o modelo MS-VAR, em que todos os parâmetros variem, permitindo assim fazer certas inferências por meio dessa flexibilização e dessas quebras.

Os dados empregados na investigação da hipótese da não linearidade entre a política monetária e o câmbio no Brasil consiste de séries de tempo mensais da economia brasileira, compreendendo o período de janeiro de 2000 a outubro de 2016, totalizando 201 observações. Excluiram-se os primeiros 6 meses de aplicação do Regime de Metas de Inflação devido ao excesso de volatilidade das variáveis aqui empregadas. São utilizadas quatro variáveis: i) Índice de variação média do preço das commodities (IC) - utilizada como medida de oferta agregada; ii) Índice de pro-

\footnotetext{
${ }^{8}$ Para maiores detalhes ver: McLachlan e Krishnan (2007).
} 
dução física (quantum) Industrial (IPI) - utilizada como medida de demanda agregada; iii) Índice de Preços ao Consumidor Amplo (IPCA); e iv) Taxa média mensal de Câmbio R \$/US\$ (TXC). Todas as variáveis são expressas em logaritmo natural, uma vez que se encontravam na forma de índice ou na forma de valores monetários.

Os dados foram extraídos da base de dados online do Instituto de Pesquisa Econômica Aplicada - IPEADATA (2017), do banco de dados de séries temporais do Banco Central do Brasil (BCB, 2017a), do Instituto Brasileiro de Geografia e Estatística (IBGE, 2017) e do Fundo Monetário Internacional (IMF, 2017). A escolha das variáveis citadas baseou-se no trabalho de Araújo e Modenesi (2010) em que o Brasil tem três determinantes macroeconômicos do nível de preços: i) demanda agregada (IC); ii) oferta agregada (IPI); e iii) taxa de câmbio (TXC). O comportamento de cada variável pode ser observado na Figura 2 a seguir:

Figura 2: Comportamento das variáveis selecionadas ao longo do tempo

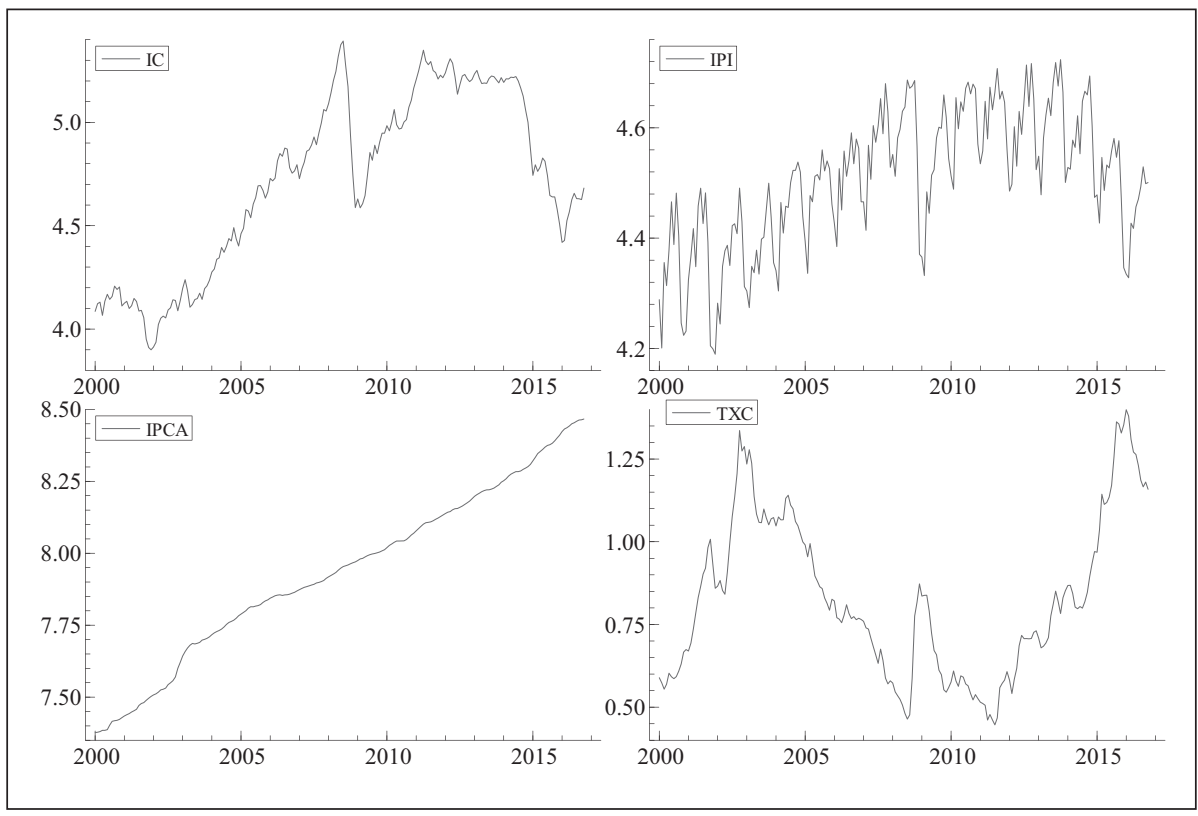

Fonte: Elaborado a partir dos dados da pesquisa

Há de se fazer uma ressalva, com relação à escolha das variáveis. A adoção da variável IPI, referente à produção industrial como proxy das condições de demanda. Como a produção industrial remonta a menos de $20 \%$ do Produto Interno Bruto (PIB), seu uso traz limitações que não podem ser ignoradas. O ideal seria a adoção de um indicador mais amplo da atividade econômica, que, além de contemplar a produção industrial, contemplasse também o setor de serviços, por exemplo. Contudo, na ausência de um índice mais fidedigno do nível de atividade econômica de periodicidade mensal, a opção pela adoção da produção industrial torna-se inevitável, o que aliás se tornou usual sua adoção pela literatura empírica, como 
mostrado nos trabalhos de Araújo e Modenesi (2010), Modenesi e Araújo (2013) e Fonseca, Peres e Araújo (2016).

Os softwares utilizados para a realização deste trabalho foram o Eviews 7.0 e o OxMetrics 7.0 com o pacote Enterprise. Feitas essas devidas considerações, parte-se a seguir para a estimação do modelo MS-VAR, que será apresentada na próxima seção.

\section{RESULTADOS E DISCUSSÃO}

Um passo importante antes de se proceder às estimações propriamente ditas se refere à realização de alguns testes que visam identificar os procedimentos e critérios para a seleção do modelo adequado. Primeiramente, apresentam-se testes de seleção de defasagem que irão indicar qual o número correto de defasagens a ser utilizado no modelo, como mostra a Tabela 4:

Tabela 4: Teste de seleção de defasagem

\begin{tabular}{c|c|c|c|c|c|c}
\hline Defasagens (p) & LogL & LR & FPE & AIC & SC & HQ \\
\hline 0 & 320.24 & NA & $4.90 \mathrm{E}-07$ & -3.178291 & -3.112094 & -3.151499 \\
\hline 1 & 1830.155 & 2943.955 & $1.48 \mathrm{E}-13$ & -18.19251 & -17.86153 & -18.05856 \\
\hline 2 & 1918.833 & 169.3347 & $7.11 \mathrm{E}-14$ & -18.92295 & $-18.32717^{*}$ & $-18.68182^{*}$ \\
\hline 3 & 1939.335 & $38.32537^{*}$ & $6.80 \mathrm{e}-14^{*}$ & $-18.96819^{*}$ & -18.10763 & -18.6199 \\
\hline
\end{tabular}

Fonte: Elaboração própria.

* Indica o número de defasagens selecionado por cada critério para o sistema VAR.

LR: estatística LR; FPE: erro final de previsão; AIC: critério de informação de Akaike; SC: critério de informação de Schwarz; HQ: critério de informação de Hannan-Quinn.

$\mathrm{Na}$ posse dessas informações, podem der usados esses dados para estimar e analisar o modelo MS-VAR. Para se observar o comportamento do nível de preços, estimou-se um modelo MS-VAR irrestrito, com intercepto, variância e parâmetros variando conforme o regime. Dessa forma, utilizando a nomenclatura desenvolvida por Krolzig (1997), estimou-se um MS(2)-VAR(1), em que o número de regimes possível $-m$, foi travado em 2 (dois) e a defasagem ótima $-p$, escolhida igual a 1 (um). A explicação do por que se fixou o número de regimes em 2 (dois) foi, portanto, supor que há dois regimes para o nível de preços, ou seja, haveria um regime de alta e outro de baixa para a taxa de inflação. Ademais, a quantidade de parâmetros a serem estimados, uma vez que, quando se estima um modelo MS-VAR com três regimes, o número de parâmetros cresce consideravelmente, tornando a estimação do modelo inviável estatisticamente. 
Quanto ao número de defasagens, a escolha difere dos testes de seleção de defasagem, uma vez que os resultados obtidos com o modelo MS(2)-VAR(1) mostrou-se mais ajustado e significativo estatisticamente. Isso quer dizer que o modelo estimado por ser mais simples, com um elevado grau de liberdade e mais ajustado tornou-se a melhor opção a ser apresentada no presente artigo. Ademais, os modelo estimados com as especificações MS(2)-VAR(2) e MS(2)-VAR(3) mostraram problemas nos seus resíduos, além da perda de graus de liberdade, uma vez que, quando se aumenta as defasagens, o número de parâmetros estimados aumenta consideravelmente, inviabilizando a estimação, dado o limitado número de observações, uma vez que a análise centra-se no período posterior a adoção do RMI. Assim, levando em consideração o teste de linearidade (Teste LR) na Tabela 5, percebe-se que o modelo em questão é não linear, a uma significância de $1 \%$, e que os parâmetros mudam de maneira significativa entre os regimes, justificando dessa forma o uso do modelo MS-VAR.

Tabela 5: Teste LR de linearidade

\begin{tabular}{l}
$\mathrm{H}^{0}-\mathrm{O}$ modelo é linear \\
Teste de linearidade LR $4967.8 \quad[0.0000]^{*}$ \\
\hline
\end{tabular}

Fonte: Elaborado a partir dos dados da pesquisa.

Quanto aos resíduos do MS-VAR estimado, estes se apresentaram bem-comportados, como mostra a Figura 3 a seguir:

Figura 3: Correlograma, densidade e QQ-Plot dos resíduos-padrão no MS-VAR.

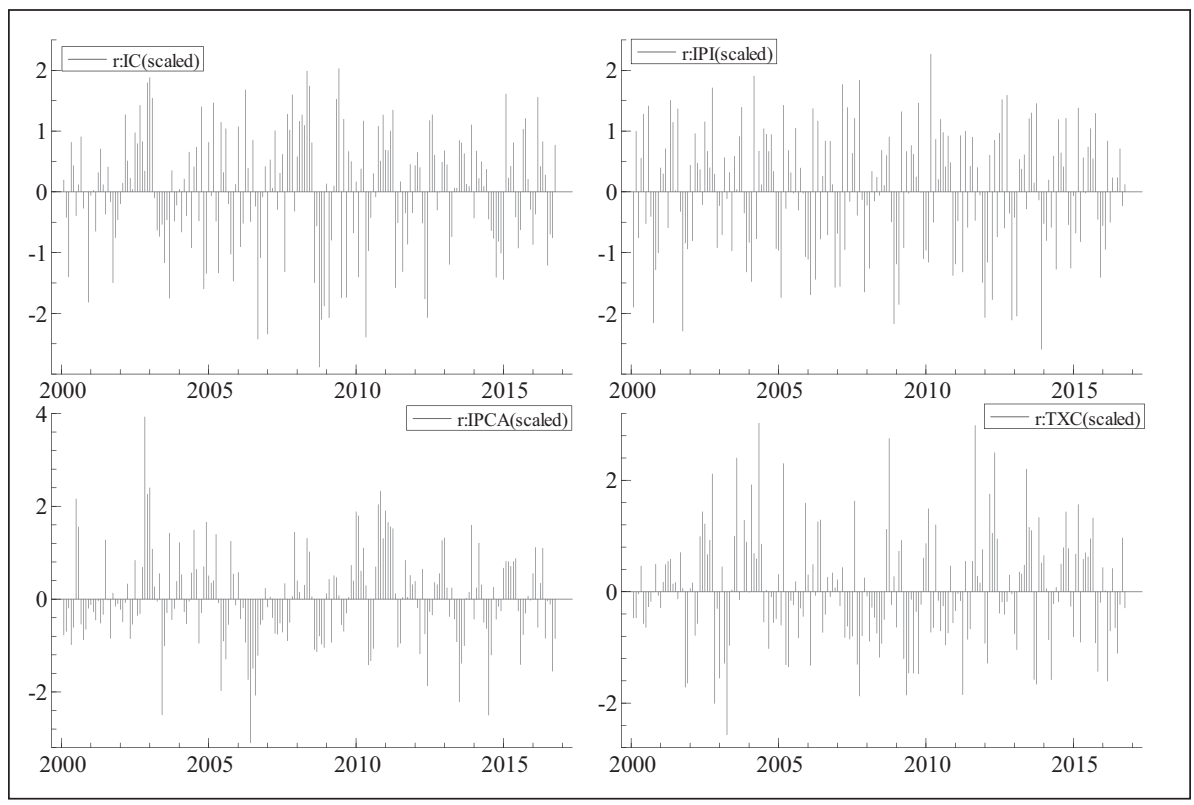

Fonte: Elaborado a partir dos dados da pesquisa 
Nos testes residuais, os resíduos apresentaram-se comportados e tendem a uma distribuição normal. O teste de normalidade dos resíduos mostrou-se significativo a 1\%. A análise gráfica dos correlogramas, densidade e o QQ-Plot dos resíduos são apresentados na Figura 4, a seguir.

Na referida Figura, os resíduos mostram-se pouco correlacionados e sua distribuição tende a ser uma distribuição normal, com exceção da variável Ind, que apresenta sua distribuição dos resíduos com uma calda alongada para a esquerda, fato que não compromete de maneira significativa o modelo estimado. Já a ferramenta QQ-Plot, é uma ferramenta relativamente simples, mas muito importante, quando se quer analisar a distribuição dos resíduos comparados a uma distribuição normal (Cleveland, 1985).

Figura 4: Correlograma, densidade e QQ-Plot dos resíduos-padrão no MS-VAR

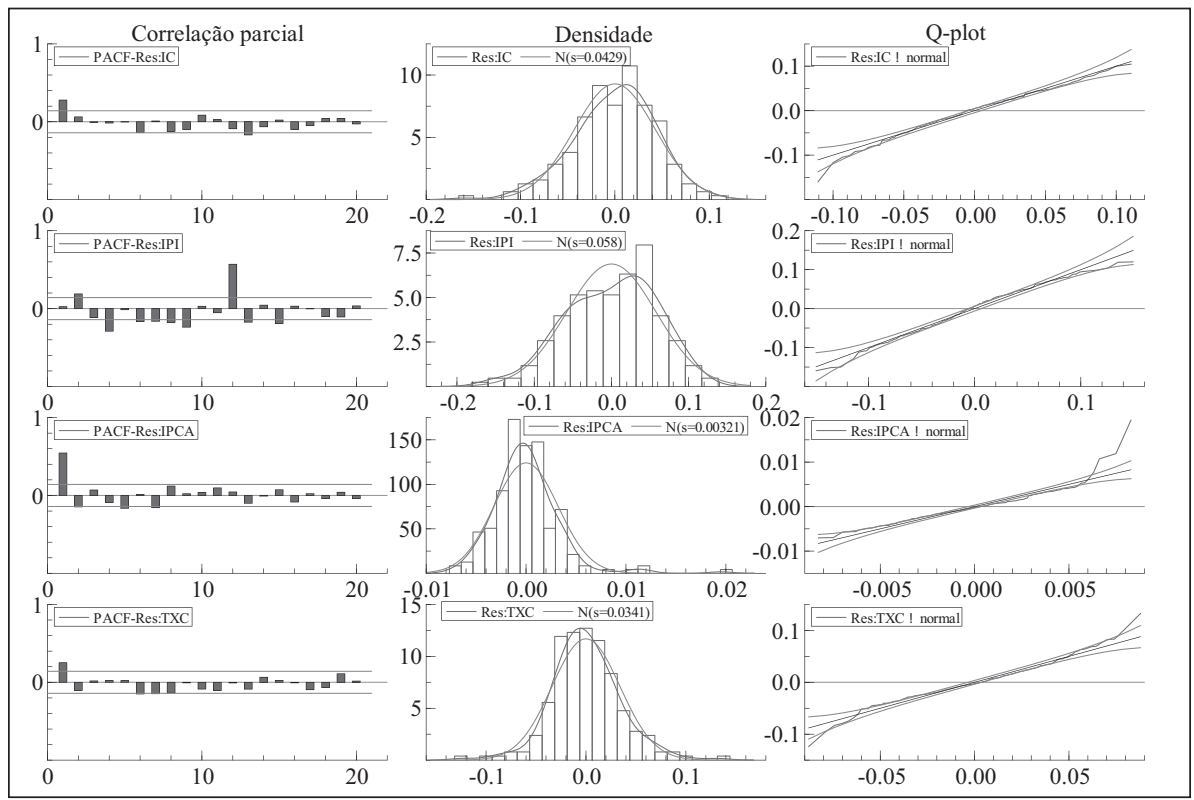

Fonte: Elaborado a partir dos dados da pesquisa.

Desta forma, pode-se verificar que os resíduos do modelo estimado têm distribuições bem-comportadas e próximas de uma distribuição normal (na média), o que sugere que, o uso de séries não estacionárias como variáveis endógenas no modelo, em nada comprometeu a estimação dos resultados.

A convergência do algoritmo EM se deu após 6 interações, com uma probabilidade de mudança de 0,0001. A Figura 5 mostra o bom ajustamento do modelo em cada regime estimado. 


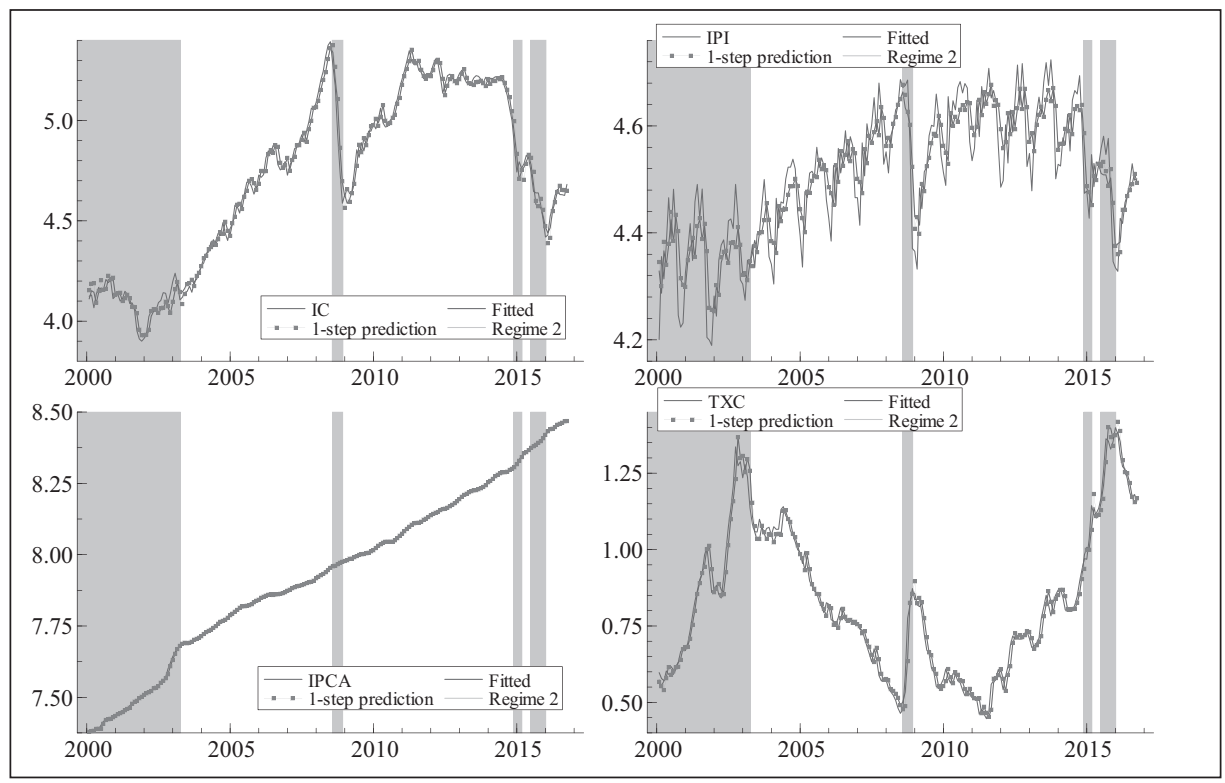

Fonte: Elaborado a partir dos dados da pesquisa.

O modelo MS(2)-VAR(1) estimado para o período de janeiro de 2000 a outubro de 2016 mostrou a seguinte matriz de transição dos regimes:

$$
\hat{T}=\left[\begin{array}{cc}
0,97276 & 0,066781 \\
0,027241 & 0,93322
\end{array}\right]
$$

Pode-se perceber, através da matriz $\hat{T}$, que os regimes estimados no modelo são persistentes, isto é, uma vez que política monetária permanece em um dos regimes, a probabilidade de conservar-se neste mesmo regime é alta. Isso porque, segundo a matriz $\hat{T}$, estando no primeiro regime, a probabilidade de mudar para o segundo regime é de apenas $2,7 \%$, enquanto que, permanecer no mesmo regime, a probabilidade é de 97,3\%. O mesmo ocorre no segundo regime; uma vez estando nele, a probabilidade de mudança é de apenas $6,7 \%$, e a de permanência é de 93,3\%.

A Figura 5 mostra que, de acordo com as probabilidades estimadas, pode-se classificar temporalmente os dois regimes, tendo como resultado a Tabela 6, na página seguinte.

O Regime 1 (um) se mostra mais persistente e o mais predominante, totalizando 146 meses do período analisado e tendo uma média de duração de 36 meses. Já o Regime 2 configura uma menor persistência, totalizando 55 meses do período analisado e tendo uma média de 14 meses de duração. No que diz respeito aos períodos dos regimes estimados, deve-se fazer duas ressalvas importantes. A primeira diz respeito à classificação estimada dos regimes. O modelo MS-VAR estima empiricamente a classificação dos regimes de maneira automática, conforme as características já supracitadas anteriormente. Dessa forma, quando se compara os 


\begin{tabular}{|c|c|}
\hline Regime 1 & Regime 2 \\
\hline 05/2003 - 07/2008 (0.998) & $02 / 2000-04 / 2003(0.994)$ \\
\hline 01/2009 - 11/2014 (0.990) & $08 / 2008-12 / 2008$ (0.908) \\
\hline 04/2015 - 06/2015 (0.948) & $12 / 2014-03 / 2015$ (0.999) \\
\hline 02/2016 - 10/2016 (0.979) & $07 / 20015-01 / 2016(0.921)$ \\
\hline Total: 146 meses & Total: 55 meses \\
\hline $\begin{array}{l}\text { Representa } 72,64 \% \text { do período estimado com } \\
\text { uma média de duração de } 36,5 \text { meses. }\end{array}$ & $\begin{array}{l}\text { Representa } 27,36 \% \text { do período estimado com } \\
\text { uma média de duração de } 13,75 \text { meses. }\end{array}$ \\
\hline
\end{tabular}

Fonte: Elaborado a partir dos dados da pesquisa.

Nota: As probabilidades estão entre parênteses.

resultados obtidos da estimação na Tabela 6 com os dados observados na tabela 2, percebem-se algumas diferenças. A primeira e mais visível está no período de 01/2009 a 11/2014, em que o modelo estimado mostra o Regime 1 e que a taxa de câmbio teve um período de apreciação até 07/2011 seguida de uma depreciação até 01/2016. Essas diferenças se devem ao fato de que o modelo estimado teve como variável explicativa o nível de preços (IPCA) e que, coincidentemente, a variável câmbio explica de maneira significativa alguns dos episódios de aumento ou redução da inflação, mas não todos os episódios. Há de se lembrar que, conforme o modelo proposto por Araújo e Modenesi (2010), as outras variáveis (IPI e IC) usadas como proxy de demanda e oferta tem seu papel para explicar também movimentos no nível de preços.

Desta forma, para analisar mais pormenorizadamente cada uma das diferenças entre os modelos estimados e a diferença de regime dentro do modelo MS-VAR, usualmente são construídas as funções de impulso-resposta. Estas são importantes na análise de séries temporais, uma vez que sumarizam as informações dos parâmetros autorregressivos estimados, assim como as variâncias e covariâncias estimadas, tornando a interpretação das mudanças entre os parâmetros muito mais evidente e fácil de ser observada.

Primeiramente, analisam-se as FIR do modelo MS-VAR, que trazem duas funções distintas: as linhas tracejadas representam a estimação dependente do Regime 1, enquanto as linhas contínuas, a dependente do Regime 2. Elas estão representadas pela figura 6 , na página seguinte.

Observando as FIR obtidas, tem-se, num primeiro momento, uma relação interessante entre taxa de câmbio e IPCA. Dado um choque na taxa de câmbio, dependendo do Regime estimado pelo modelo MS-VAR, obtiveram-se diferentes respostas no nível de preços. Considerando o Regime 1, que se refere à apreciação cambial, o IPCA se comportou de maneira contracionista, isto é, apreciações cambiais fizeram com que o nível de preços se reduzisse. 
Figura 6: Função de Impulso-Resposta dependente do Regime 1

(Choque de um ponto percentual nas variáveis IC, IPI, IPCA e TXC)

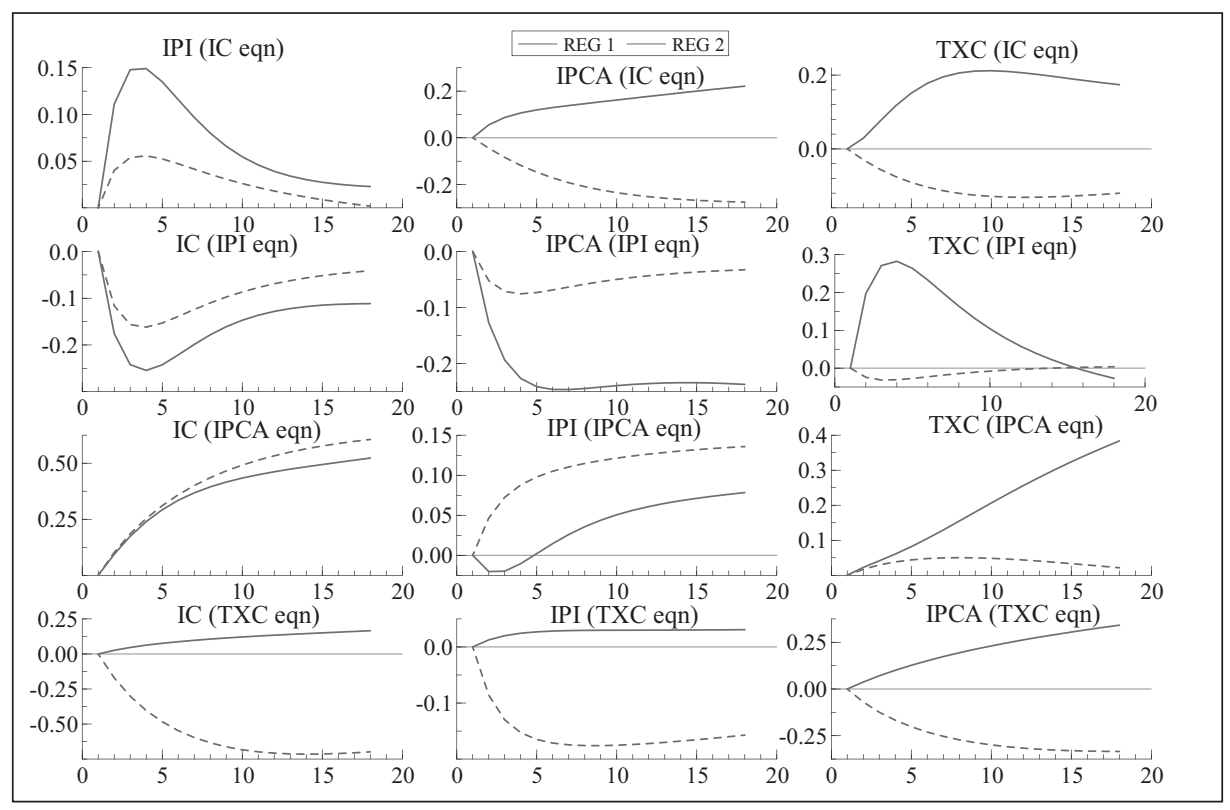

Fonte: Elaborado a partir dos dados da pesquisa.

Em contrapartida, no Regime 2, em que se observaram episódios de depreciação cambial, o IPCA teve um comportamento de alta, demonstrando assim a existência de repasse cambial independente do regime de alta ou de baixa do câmbio. Ademais, tanto pelo lado da oferta (IC) como pelo lado da demanda (IPI), há diferenças dos impactos no nível de preços dependendo do regime analisado. No Regime 1, de apreciação cambial, as FIR mostram que há uma resposta negativa do IPCA, tanto para IC quanto para IPI. No entanto, no Regime 2, o IPCA respondeu de maneira positiva ao choque no IC e com uma resposta mais negativa ao IPI.

Esse resultado é parecido com o encontrando por Squeff (2009), mas com a diferença de que o referido autor estimou as magnitudes dos repasses cambiais conforme recortes temporais arbitrados por ele. Já neste trabalho, utiliza-se da metodologia MS-VAR para estimar endogenamente, por meio de regimes, momentos de apreciação e depreciação do câmbio, demonstrando-se como os níveis de preços (IPCA)são influenciados pelos movimentos nas taxas de câmbio.

O importante a destacar, contudo, é que tais resultados demonstram um aspecto relevante sobre a política monetária brasileira, esclarecendo que, em períodos em que a moeda brasileira se encontra em trajetória de apreciação, tanto pelo lado da demanda quanto da oferta, existe um conjunto de forças que determinam uma trajetória baixista aos níveis de preços, contribuindo com a política monetária, já que torna maior a possibilidade das Autoridades Monetárias serem bem-sucedidas no cumprimento das metas de inflação estabelecidas. 
Em assim sendo, a conclusão a que se chega é a de que, se realmente o canal do câmbio se mostra um canal predominante para a transmissão da política monetária, deve-se reavaliar o modus operandi da condução da política macroeconômica, uma vez que a utilização da taxa de juros básica - Selic, como principal instrumento de atuação, no contexto de Regime de Metas de Inflação, pode não ser eficiente, e a introdução de mecanismos que venham ao encontro do gerenciamento da taxa de câmbio, elemento central para a maior eficiência da referida política. Na seção 5, a seguir, faz-se uma discussão sobre a política cambial no Brasil, bem como das implicações que advêm do fato da política cambial ser utilizada como uma ferramenta para o controle de preços.

\section{UMA DISCUSSÃO ALTERNATIVA DA POLÍTICA CAMBIAL PARA ALÉM DO CONTROLE DE PREÇOS}

A abertura da conta financeira, implementada no Brasil em 1992, permitiu que os agentes econômicos nacionais pudessem adquirir ativos e passivos denominados em moeda estrangeira e, ainda, os não residentes pudessem operar nos mercados financeiros domésticos 9 . Isso possibilitou a criação de condições para entrada e saída de capitais no país.

O cenário presente na economia brasileira desde a implementação do RMI, em 1999, caracterizou-se, portanto, por um ambiente no qual a abertura financeira implicou renúncia à administração da conta de capitais, deixando o país sujeito às oscilações dos fluxos financeiros. Os condicionantes domésticos (destaque para os altos e crescentes níveis da taxa Selic) aliados aos internacionais (o nível de liquidez internacional) promovem um influxo de capitais na economia brasileira. A taxa de câmbio - dita não administrada - tornou-se extremamente apreciada.

Sendo assim, a causa da apreciação cambial no Brasil, além da esperada, é explicada sobremaneira pela política monetária, que não acompanhou, proporcionalmente, a redução da percepção de risco-país, gerando espaço para arbitragem, com baixo risco, pelo diferencial de taxas de juros interna e externa. Isto pressionou o mercado de câmbio na direção de uma apreciação e esse patamar do câmbio no Brasil contribuiu para manter a inflação baixa.

Isso porque o câmbio apreciado, como já demostrado anteriormente, é um dos principais canais de transmissão da política monetária no Brasil. Sua atuação sobre os preços ocorre via redução dos preços das importações, sejam matérias-primas ou produtos finais. As matérias-primas diminuem os preços finais de produtos nacionais que as usam como insumos e os produtos finais importados pressionam para baixo os preços dos bens nacionais concorrentes. Outra questão importante é a influência que o dólar baixo exerce sobre preços administrados, como da energia elétrica, telefonia e planos de saúde, por exemplo. Esses preços têm seus reajus-

\footnotetext{
${ }^{9}$ Para uma ampla discussão a respeito da liberalização financeira no Brasil, ver Sicsú (2007).
} 
tes dados pela variação do Índice Geral de Preços do Mercado (IGP-M), que é fortemente influenciado pelo dólar.

No entanto, há que se ressaltar que um processo de apreciação cambial provoca perda de competitividade da indústria nacional, contribui aos déficits em transações correntes e a primarização da pauta exportadora. Essas consequências negativas estão na contramão daqueles que defendem a importância da taxa de câmbio como uma estratégia de desenvolvimento econômico.

Williamson (2003) foi um dos primeiros autores a defender a abordagem da taxa de câmbio como estratégia de desenvolvimento. Sua análise apoia-se em Balassa (1982), que descreve o êxito das economias da Ásia Oriental - inicialmente, Hong Kong, Coreia, Cingapura e Taiwan, e, em seguida, os países do Sudeste Asiático - em sustentar altas taxas de crescimento econômico, mediante a adoção de taxas de câmbio competitivas. A maioria destas economias passou por uma fase inicial de industrialização por substituição de importação e, ao invés de manterem essa estratégia a um ponto em que fossem obrigadas a construir sofisticadas indústrias para as quais a sua dotação de fatores não estava adaptada, elas se dedicaram à exportação de produtos manufaturados simples. Ao longo do tempo, a fabricação básica expandiu-se e esses países começaram a produzir e a exportar uma gama maior de produtos manufaturados. Além disso, em virtude do rápido crescimento de suas exportações, estas economias convenceram os mercados de que eram bons destinos para os fluxos de capitais, em especial para os investimentos estrangeiros diretos.

No entanto, Williamson (2003) ressalta que, a despeito do importante papel que a taxa de câmbio real tem na economia, os países em desenvolvimento não têm enfatizado tal política. As discussões a esse respeito concentram-se, em geral, sobre problemas macroeconômicos de curto prazo, enquanto a análise de longo prazo é focada mais na sustentabilidade externa do que no crescimento econômico e no emprego.

Também reforçando a defesa da abordagem da taxa de câmbio como estratégia para o desenvolvimento, Frenkel (2004) analisa a relação entre taxas de câmbio real e emprego na Argentina, no Brasil, no Chile e no México. Conforme o autor, existem diversas experiências com políticas cambiais no período posterior à liberalização financeira nesses países. Muitas delas focam, primariamente, o controle da inflação, isto é, as taxas de câmbio são utilizadas como âncoras para a estabilização, como foi o caso da Argentina e do Chile nos anos 1970, do México nos anos 1980 e 1990, e da Argentina e do Brasil nos anos 1990. Em todas essas experiências, as políticas adotadas levaram a crises econômicas. Mais recentemente, regimes de taxas de câmbio flutuantes foram adotados, embora as políticas macroeconômicas continuassem, na maioria das vezes, focando sempre o problema da inflação em detrimento das metas reais, como crescimento e emprego, que foram excluídos do foco principal das políticas cambiais.

Ao analisar a política cambial desses países entre 1980 e 2003, Frenkel (2004) conclui que preservar a competitividade e a estabilidade da taxa de câmbio real é uma das melhores contribuições que a política macroeconômica pode dar para melhorar o desempenho macroeconômico nos países em desenvolvimento. Sendo 
assim, a política cambial deve ser apresentada como um componente de um regime de política macroeconômica apto a perseguir diversos objetivos - na maioria das vezes conflitantes - em um caminho coerente, que considere o crescimento e o desenvolvimento econômico de longo prazo.

Rodrik (2004 e 2008) também defende a importância da política cambial como uma estratégia de crescimento. Ele entende que, para uma pequena economia aberta, a taxa de câmbio real é definida como a relação entre o preço dos produtos comercializáveis e não comercializáveis com o exterior. Na prática, esse preço tende a se mover simultaneamente com a taxa de câmbio nominal. Assim, se ocorrer uma desvalorização da moeda (apoiada por políticas monetárias e fiscais adequadas), isso implicará aumento da lucratividade das atividades comercializáveis com o exterior. Essa desvalorização, explica o autor, tem uma série de vantagens. Uma delas é o início da diversificação das atividades dos países em direção a atividades não tradicionais. Outra vantagem é que as atividades comercializáveis se deparam com a concorrência externa, enfrentando maior disciplina de mercado, o que as torna mais dinâmicas comparadas com as demais. Por fim, a maneira pela qual a depreciação subsidia atividades comercializáveis é completamente amigável ao mercado, no sentido de que não requer nenhuma espécie de microgestão por parte dos burocratas. Baseado nessas razões, o autor defende que uma política crível e sustentada de desvalorização da taxa de câmbio real constitui uma das formas mais eficazes de política industrial.

Rodrik (2004) ressalta ainda que altas taxas de câmbio reais têm desempenhado um importante papel em alguns dos processos mais recentes de aceleração do crescimento. Por exemplo, isso foi o que aconteceu com o Chile, desde meados dos anos 1980, quando uma grande oscilação dos preços relativos em favor dos bens comercializáveis acompanhou o crescimento desse país. Mais que uma duplicação da taxa de câmbio real, na sequência da crise chilena de 1982-1983 desempenhou um papel fundamental para promover a diversificação das exportações não tradicionais e estimular o crescimento econômico.

Por fim, a abordagem da taxa de câmbio como estratégia de desenvolvimento recebe respaldo de Bresser-Pereira (2007) e Bresser-Pereira et al. (2014), que asseveram que, embora a taxa de câmbio seja um dos principais preços da economia, permanece ainda pouco estudada e mal compreendida. Destacam os autores que, nos países em desenvolvimento, a taxa de câmbio é essencialmente uma ferramenta de crescimento econômico. Primeiro, porque uma taxa de câmbio desvalorizada assegura ao país a estabilidade do balanço de pagamentos. Segundo, e sobretudo, porque taxas de câmbio competitivas aumentam a poupança e o investimento.

No que se refere à primeira razão, a taxa de câmbio competitiva estimula exportações e restringe importações, mantendo o equilíbrio em conta-corrente. Quanto à segunda, a taxa de câmbio competitiva mantém os salários baixos e, como o consumo agregado varia de acordo com o total de salários, este também sofre redução. Por outro lado, a taxa de câmbio competitiva cria oportunidades para investimentos em indústrias exportadoras, promovendo elevação dos investi- 
mentos e do Produto Interno Bruto (PIB), o que compensa a repressão inicial do consumo.

De acordo com os autores essa é uma das explicações para o sucesso das economias do Leste Asiático. Elas mantiveram a taxa de câmbio desvalorizada de modo a atingir altas taxas domésticas de poupança e de investimento e para evitar crises de balanço de pagamentos.

Em suma, as análises mencionadas anteriormente ressaltam que a manutenção da taxa de câmbio em níveis competitivos pode estimular o crescimento econômico, destacando experiências bem-sucedidas de países que utilizaram tal estratégia em contraste com países que se devotaram à política cambial para solucionar problemas inflacionários.

\section{COMENTÁRIOS FINAIS E IMPLICAÇÕES DE POLÍTICAS}

Este artigo analisou, com base em um modelo Markov Swichting Vector Autoregressive (MS-VAR) evidências de não linearidade da relação entre câmbio e preços no Brasil. Nessa análise demonstrou-se que o canal do câmbio é um mecanismo central para a transmissão da política monetária, tanto em momentos de depreciação, como também e principalmente, nas fases de apreciação cambial quando, tanto as variáveis do lado da oferta, quanto do lado da demanda, apresentam trajetória que contribuem para a redução do nível geral de preços.

Tal fato sugere que a taxa de câmbio tem um papel fundamental no controle da inflação e no desempenho do próprio RMI, todavia, apontam para a necessidade de uma reavaliação da política cambial que vem sendo adotada no Brasil para além do papel de mecanismo de controle de preços. A experiência internacional inclusive tem demostrado, nesse sentido, que diversas experiências bem-sucedidas de crescimento econômico têm utilizado a taxa de câmbio competitiva como importante ferramenta para estimular o crescimento e o desenvolvimento econômico.

\section{REFERÊNCIAS BIBLIOGRÁFICAS}

Akerlof, G. A., Blanchard, O., Romer, D., \& Stiglitz, J. E. (Eds.). (2014). What have we learned?: Macroeconomic policy after the crisis. Cambridge (MA): MIT Press.

Araújo, E. C. Modenesi, A. M. (2010). A Importância do Setor Externo na Evolução do IPCA (19992010): uma análise com base em um modelo SVAR. In: Anais XXXVIII Encontro Nacional de Economia (ANPEC), Salvador (BA).

Balassa, B. A. (1982). Development strategies in semi-industrial economies. Published for the World Bank [by] the Johns Hopkins University Press.

Banco Central Do Brasil (BCB). (2017a). Séries Temporais. http://www.bcb.gov.br. Acesso em março de 2017.

Banco Central Do Brasil (BCB). (2017b). Boletim do Banco Central. Relatório Anual 2008-2009. Disponível em: http://www.bcb.gov.br. Acesso em março de 2017.

Belaisch, M. A. (2003). "Exchange rate pass-through in Brazil”. Working Paper n. 3-141. International Monetary Fund (IMF). 
Bresser-Pereira, L. C.; Oreiro, J. L.; Marconi, N. (2014). Developmental Macroeconomics: New Developmentalism as a Growth Strategy. New York: Routledge.

Bresser-Pereira, L. C. (2007). Macroeconomia da estagnação: crítica da ortodoxia convencional no Brasil pós-1994. São Paulo: Editora 34.

Cleveland, W. S. (1985). The elements of graphing data. Monterey (CA): Wadsworth advanced books and software, 1985.

Diaconis, P.; Stroock, D. (1991). “Bounds for Eigenvalues of Markov Chains”. The Annals of Applied Probability, 1(1), 36-61.

Ehrmann, M.; Ellison, M.; Valla, N. (2003). "Regime-dependent impulse response functions in a Markov-switching vector autoregression model”. Economics Letters, 78(3), 295-299.

Fonseca, M. R. R. Da; Peres, S. C; Araújo, E. C. de. (2016). "Regime de metas de inflação: análise comparativa e evidências empíricas para países emergentes selecionados". Revista de Economia Contemporânea, 20(1), 113-143.

Frenkel, R. (2004) Real exchange rate and employment in Argentina, Brazil, Chile and Mexico. Buenos Aires: CEDES, paper presented to the G24. Disponível em: < https://goo.gl/hz57aH >. Acesso em: mar. 2017.

Hamilton, J. D. (1989). "A new approach to the economic analysis of nonstationary time series and the business cycle”. Econometrica: Journal of the Econometric Society, 357-384.

Instituto Brasileiro de Geografia e Estatística (IBGE) (2017). Sistema Nacional de Índices de Preços ao Consumidor (IBGE/SNIPC). Disponível em: < https://goo.gl/3rDKNJ >. Acesso em: mar. 2017.

Instituto De Pesquisa Econômica Aplicada - IPEADATA (2017). Dados macroeconômicos e regionais. Disponível em: <http://www.ipeadata.gov.br>. Acesso em: 3 mar. 2017.

International Monetary Fund. (IMF) (2017). World Economic Outlook Database. October 2016. Disponível em: < https://goo.gl/MhpNvM >. Acesso em: 22 de março de 2017.

Kannebley Jr, S. (2000). "Exchange rate pass-through: uma análise setorial para as exportações brasileiras (1984-1997)". Economia Aplicada, 4(3), 435-463.

Krolzig, H. M. (1996). Statistical analysis of cointegrated VAR processes with Markovian regime shifts. SFB 373 Discussion Paper 25/1996. Humboldt-Univ., Wirtschaftswiss. Fak.

Krolzig, H. M. (1997). Markov-Switching Vector Autoregressions: Modelling, Statistical Inference, and Application to Business Cycle Analysis. Berlin (Germany): Springer-Verlag Berlin Heidelberg.

Krolzig, H. M. (1998). Econometric modelling of Markov-switching vector autoregressions using MSVAR for Ox. unpublished, Nuffield College, Oxford. Disponível em: < https://goo.gl/572nZ8 >. Acesso em: mar. 2017.

Mclachlan, G.; Krishnan, T. (2007). The EM Algorithm and Extensions. New York: J. Willey \& Sons.

Modenesi, A. De M.; Araujo, E. C. de (2013). "Price stability under inflation targeting in Brazil: empirical analysis of the mechanism of transmission of the monetary policy based on a VAR model, 20002008". Investigación Económica, 72(283), 99-133.

Menon, J. (1995). “Exchange rate pass-through”. Journal of Economic Surveys, 9(2), 197-231.

Minella, A.; Correa, A. S. (2005). Mecanismos não-lineares de repasse cambial: um modelo de Curva de Phillips com Threshold para o Brasil. In: Anais XXXIII Encontro Nacional de Economia (ANPEC), Natal (RN).

Minella, A., de Freitas, P. S., Goldfajn, I., \& Muinhos, M. K. (2003). "Inflation targeting in Brazil: constructing credibility under exchange rate volatility". Journal of international Money and Finance, 22(7), 1015-1040.

Neal, R. M. (1993). Probabilistic Inference Using Markov Chain Monte Carlo Methods, Technical Report CRG-TR-93-1, Department of Computer Science, University of Toronto. Disponível em: < https://goo.gl/nXgjLR >. Acesso em: abr. 2017.

Rodrik, D. (2004). Growth strategies. John F. Kennedy School of Government, Harvard University, Draft, August. Disponível em: < https://goo.gl/V88gLi >. Acesso em: abr. 2017.

Rodrik, D. (2008). The Real Exchange Rate and Economic Growth: Theory and Evidence. Disponível em $<$ https://goo.gl/Yjq7F6 > . Acesso em: abr. 2017. 
Sims, C. A. (1990). "Solving the stochastic growth model by backsolving with a particular nonlinear form for the decision rule". Journal of Business \& Economic Statistics, 8(1), 45-47.

Sicsú, J. (2007). Emprego, Juros e Câmbio: Finanças Globais e Desemprego. Ed. Campus, Rio de Janeiro. Squeff, G. C. (2009). Repasse cambial "reverso": uma avaliação sobre a relação entre taxa de câmbio e IPCA no Brasil (1999-2007). Dissertação (Mestrado). Programa de Pós-Graduação em Ciências Econômicas. Universidade Estadual do Rio de Janeiro, 106 p.

Tombini, A. A.; Alves, S. A. L. (2006). The recent Brazilian disinflations process and costs. Working Papers Series $n^{\circ} 109$. Brasília: Banco Central do Brasil.

Williamson, J. (2003) Exchange rate policy and development. Presented in Initiative for Policy Dialogue Task Force on Macroeconomics, Columbia, New York. 